\title{
Afirmaciones epistemológicas con "alta carga teórica" que pueden tener incidencia en la didáctica de las ciencias: un estudio comparativo
}

\author{
Philosophy of science propositions with \\ "high theory-ladenness" that may have an incidence \\ in science education: a comparative study
}

\author{
Rafael Yecid Amador-Rodríguez ${ }^{1}$. Agustín Adúriz-Bravo²
}

\begin{abstract}
Resumen: Asumiendo la naturaleza de la ciencia (NOS) como un conjunto de contenidos metateóricos con valor para la educación científica, entendemos que en este campo se priorizan modelos provenientes la epistemología, con el objeto de que la ciudadanía genere respuestas a las cuestiones de qué es la ciencia, cómo se transforma a lo largo del tiempo y cómo se relaciona con su contexto social y cultural. El propósito de este documento es recoger y comparar algunas de las que llamamos "afirmaciones con alta carga teórica" en relación con la epistemología presentadas en documentos especializados que son fuente de consulta para algunos integrantes de la comunidad de didáctica de las ciencias ocupados de la NOS.
\end{abstract}

Palabras clave: Didáctica de las ciencias. Epistemología. Naturaleza de la ciencia. Filosofía de la ciencia.

\begin{abstract}
Assuming the nature of science (NOS) as a set of items of meta-theoretical content with value for science education, we understand that in this area the prioritised models are generated in the philosophy of science, with the aim of helping citizens to generate answers around the issues of what science is, how it changes in time, and how it relates to its social and cultural context. The purpose of this document is to collect and compare some of what we call 'propositions with high theory-ladenness' in relation to the philosophy of science presented in specialised documents that are references for some members of the community of science education working in NOS.
\end{abstract}

Keywords: Philosophy of science. Epistemology. Nature of science. Didactics of science.

\footnotetext{
${ }^{1}$ Instituto de Investigaciones Centro de Formación e Investigación en Enseñanza de las Ciencias (CeFIEC), Facultad de Ciencias Exactas y Naturales, Universidad de Buenos Aires. $2^{\circ}$ Piso, Pabellón 2, Ciudad Universitaria, Avenida Intendente Güiraldes 2160, (C1428EGA) Ciudad Autónoma de Buenos Aires, Argentina. E-mail: rafaelyecid@gmail.com

${ }^{2}$ Instituto de Investigaciones Centro de Formación e Investigación en Enseñanza de las Ciencias (CeFIEC), Facultad de Ciencias Exactas y Naturales, Universidad de Buenos Aires.
} 


\section{Introducción}

Uno de los aspectos por los cuales la didáctica de las ciencias se constituye en una disciplina científica reconocible es por el surgimiento y consolidación de sus "campos" de especialidad; uno de los más recientes de esos campos es el de la naturaleza de la ciencia, o NOS (por las siglas del inglés "nature of science"). Se comprende este campo como una "arena híbrida fértil" en la que se intersecan conocimientos de epistemología, historia y sociología de la ciencia (LEDERMAN, 1992; MCCOMAS, 1998). Entendemos la NOS como un área que emerge a partir de las necesidades que se establecen en los distintas niveles educativos en pos de generar una alfabetización científica para todos y todas, en la que se combinen dos aspectos importantes: saber ciencias y saber sobre ciencias (AMADOR-RODRÍGUEZ; ADÚRIZBRAVO, 2009).

En este documento se presentan los resultados cualitativos de un análisis bibliográfico hecho sobre un pequeño corpus de producciones provenientes de la epistemología y la didáctica de las ciencias recientes. Tales producciones se seleccionaron porque presentan periodizaciones explicitas de la epistemología del siglo XX, es decir, los documentos escogidos compilan argumentos relevantes y específicos correspondientes a la actividad científica y que se ajusta a la periodización que hemos propuesto para la selección de las ACT's, lo que nos permite no recurrir a los trabajos originales, ya que estos documentos presentan los marcos teóricos más relevantes de cada época epistemológica como también por su finalidad didáctica (por ejemplo, para discriminar entre diferentes versiones de método científico o para mostrar la evolución del concepto de teoría). Desde la propia didáctica de las ciencias, se han realizado propuestas de periodizaciones al respecto (ver ARIZA et al., 2009); para los fines de este trabajo nos basamos en la propuesta hecha por uno de nosotros que sitúa las aportaciones epistemológicas a la didáctica en tres "épocas": 1. positivismo lógico y concepción heredada; 2 . nueva filosofía de la ciencia y racionalismo crítico; y 3. posmodernismo y visiones contemporáneas. El propósito de nuestro estudio es el de identificar y caracterizar lo que llamamos afirmaciones con una alta carga teórica (ACT's), con el objeto de reconocer posibles aportaciones epistemológicas al campo de la naturaleza de la ciencia (NOS).

\section{Descripción del trabajo}

Para realizar el estudio, se seleccionaron cinco (5) textos (ver cuadro 1) que presentan un desarrollo epistemológico explícito, es decir, que dicen algo acerca de la naturaleza del conocimiento científico. De acuerdo con la periodización que seleccionamos para el trabajo, intentamos ubicar los planteamientos epistemológicos de la muestra en tres grandes "grupos": planteamientos que sintonizan con el positivismo lógico y la concepción heredada, planteamiento que sintonizan con la nueva filosofía de la ciencia y el racionalismo crítico, y planteamientos que se podrían inscribir en una posición contemporánea de la epistemología, la denominada concepción semántica de las teorías científicas. Nos interesa particularmente esta época porque como lo señala Izquierdo (2000), muchos de los planteamientos teóricos surgidos en la tercera época de la epistemología son de una gran riqueza para la didáctica de las ciencias, puesto que permiten generar relaciones con mayor poder teórico y metodológico entre la filosofía de la ciencia, la psicología cognitiva y la pedagogía. 
Algunas de las características que han propuesto los especialistas por ejemplo AdúrizBravo, Izquierdo y Estany (2002) para agrupar los modelos epistemológicos pertenecientes a la época denominada postmodernimso y visiones contemporáneas serían:

- Rechazo directo al concepto de racionalidad categórica.

- Recuperación de algunos aspectos (propios de la filosofía de la ciencia clásica) tales como la relación entre realidad y predicación, o la reconstrucción racional del conocimiento científico desde una perspectiva puramente semántica, específicamente modelada desde el concepto de modelo.

En el Cuadro 1, se muestra los textos que se utilizaron para el estudio y los códigos respectivos de cada texto de donde se obtuvieron las afirmaciones con una alta carga teórica respecto de la epistemología (ACT's).

Cuadro 1. Referencia de los textos consultados para la investigación

\begin{tabular}{|c|c|}
\hline $\begin{array}{c}\text { Código } \\
\text { del texto }\end{array}$ & Referencia del texto \\
\hline AABmo & $\begin{array}{r}\text { Adúriz-Bravo, A. Concepto de modelo científico: una mirada epistemológica de su evolución, } \\
\text { en Galagovsky, L. (coord.). Didáctica de las ciencias naturales: El caso de los modelos } \\
\text { científicos. Buenos Aires: Lugar Editorial, 2011. p. 141-161. }\end{array}$ \\
\hline AABme & $\begin{array}{r}\text { Adúriz-Bravo, A. ¿Existirá el "método científico"?. En: Galagovsky (coordinadora). ¿Qué tienen } \\
\text { de "naturales" las ciencias naturales. Buenos Aires: Editorial Biblos, 2008. p. 47-59. }\end{array}$ \\
\hline DC & Díez Calzada, J. A. La concepción semántica de las teorías científicas. Éndoxa: Series \\
Filosóficas, UNED, Madrid, n. 8, p. 41-91, 1997.
\end{tabular}

Fonte: elaborado pelos autores.

Entenderemos por afirmaciones con una alta carga teórica respecto de la epistemología (ACT's) como aquellas proposiciones semánticamente potentes que describen o refieren a aspectos epistemológicos que están involucrados con la ciencia (teórico, metodológico, axiológico, etc). La unidad de análisis que nos hemos creado posee un análogo con el término de "carga teórica" que proviene de la epistemología, recordemos que una de las dificultades que tenían los representantes de la filosofía analítica y positivista de la ciencia era la dicotomía entre teoría y observación, la solución que propusieron algunos epistemólogos como por ejemplo Popper es que la visión que realiza un individuo es una acción que lleva una carga teórica, es decir, la observación de un sistema está moldeada por un conocimiento previo del sistema observado (ECHEVERRÍA, 1998), lo que para fines de este trabajo, nos permite pensar que las afirmaciones (ACT's) que presentan los autores en los textos se elaboran desde representaciones del mundo y las cuales tienen una correspondencia con una época epistemológica en concreto.

La acción metodológica fue la de seleccionar las afirmaciones textuales con una alta carga teórica (ACT's), de los textos seleccionados, al leer y analizar los documentos objeto de 
estudio, se eligieron las afirmaciones correspondientes a cada época, las cuales se presentan a continuación. A partir de las ACT's, se procedió a sintetizar las ideas más importantes al respecto. Durante la recolección de los datos se obtuvo que no todos los documentos analizados suministraron insumos al estudio, es decir, no todos los textos poseen ACT's que se puedan seleccionar y enmarcar en una época epistemológica, específicamente en la de postmodernismo y visiones contemporáneas, por ejemplo, el documento con código MJ no contribuye con ACT's a la época en mención.

\section{Positivismo lógico y concepción heredada}

En el Cuadro 2 se muestran las ATC's que "sintonizan con" es decir, se nos aparecen como teóricamente afines a) los planteamientos teóricos del positivismo lógico y la concepción heredada. Para la organización de la información se formuló el criterio denominado "unidad estructural del conocimiento", el cual remite al término más importante para la comunidad científica y del sería el objetivo de la actividad científica.

Cuadro 2. ACT's correspondientes al positivismo lógico y concepción heredad

\begin{tabular}{|c|c|c|c|}
\hline \multicolumn{2}{|c|}{ Época } & Positivismo lógico y concepción heredada & Documento \\
\hline \multirow{4}{*}{ Criterio } & \multirow{4}{*}{$\begin{array}{c}\text { Unidad } \\
\text { estructural } \\
\text { del } \\
\text { conocimiento }\end{array}$} & $\begin{array}{l}\text {...Las teorías empíricas de las ciencias fácticas (con } \\
\text { contenido) son cálculos interpretables (en el sentido de la } \\
\text { lógica formal), p. } 146 \ldots\end{array}$ & AABMod \\
\hline & & $\begin{array}{l}\text {..Las teorías como: a) conjuntos de enunciados } \\
\text { organizados axiomáticamente y b) sistemas axiomáticos } \\
\text { empíricamente interpretados, p. } 8 \ldots\end{array}$ & PL \\
\hline & & $\begin{array}{l}\text {... las teorías empíricas como cálculos axiomáticos } \\
\text { parcialmente interpretados, p. } 45 \ldots \text { [... [ ... Las leyes de la } \\
\text { teoría (aquellas leyes que no se deducen de otras) son los } \\
\text { axiomas, los enunciados básicos primitivos de la teoría, p. } \\
45 \ldots \text { [ ] ...la concepción clásica es calificada de sintáctica } \\
\text { por su caracterización de las teorías como conjuntos de } \\
\text { enunciados y por su énfasis general en los aspectos } \\
\text { lingüístico-sintácticos, p. } 47 \ldots \text { [ ] ... una teoría es una clase } \\
\text { de axiomas, y si nos tomamos eso en serio ello implica que } \\
\text { toda diferencia en axiomas supone una diferencia de } \\
\text { teorías, p. } 47 \ldots\end{array}$ & DC \\
\hline & & $\begin{array}{l}\text {... las orientaciones positivistas consideran que la ciencia } \\
\text { es un cuerpo de conocimiento, formado por hechos y } \\
\text { teorías que se consideran verdaderos, en el sentido de } \\
\text { estar contrastados con los datos observacionales, p. } \\
345 \ldots \text { [ ] ... El cambio de teorías y el progreso científico se } \\
\text { producen cuando existe una verificación experimental de } \\
\text { las nuevas teorías, a través de la aplicación de una } \\
\text { metodología científica objetiva y universal, p. } 345 \ldots \text { [ ] ... El } \\
\text { conocimiento se prueba por verificación positiva y las } \\
\text { nuevas teorías se adoptan como verdaderas, p. 346... [ ] } \\
\ldots \text { La verificación se realiza a través del método científico } \\
\text { empirista. Primacía de la observación, p. } 346 \ldots\end{array}$ & MJ \\
\hline
\end{tabular}

Fonte: elaborado pelos autores. 
Como se observa en el Cuadro 2, solo aparecen referenciados cuatro documentos de los cinco analizados; entendemos que la información que se presenta en el texto no referenciado no genera un aporte relevante para esta categoría de análisis.

Desde la propuesta del Círculo de Viena y la concepción heredada, se afirma que las teorías empíricas de las ciencias naturales se formulan con un grado de complejidad rígido y limitado; las teorías se entienden como un conjunto de enunciados organizados axiomáticamente (LORENZANO, 2001). El proceso de teorización "duro" se propicia en el contexto de justificación y es en el cual a un científico se le ocurren los distintos conceptos, hipótesis, teorías, etc. (DÍEZ CALZADA, 1997). Por ejemplo, se afirma que desde las sugerencias positivistas se considera que la ciencia es un cuerpo de conocimiento, constituido por hechos y teorías que se piensan verdaderos, por estar contrastados por los datos observacionales (MELLADO JIMÉNEZ, 2003).

Tal concepción del conocimiento científico es usualmente calificada de sintáctica, por caracterizar las teorías como conjuntos de enunciados lógico-lingüísticos, siendo sistemas axiomáticos empíricamente interpretados. Se afirma que los integrantes de lo que se denomino la concepción heredada concebían las teorías empíricas como cálculos axiomáticos parcialmente interpretados (DÍEZ CALZADA, 1997). Uno de los problemas que presenta los referentes teóricos de la concepción heredada es el relativo a la vinculación de los conceptos teóricos con la experiencia, sostienen los integrantes de esta postura que ese vínculo se establece a través de enunciados o reglas de correspondencia, que conectan términos teóricos con términos que, pretendidamente, refieren a entidades directamente observables, lo que actualmente la comunidad ha refutado. Se establecieron dos tipos de contextos, para los integrantes de estas posturas, la importante era la de justificación y no la de descubrimiento, la cual está relacionada con la forma como a un hombre o mujer de ciencia se la ocurre un concepto, teoría o modelo científico independientemente de cómo se le ocurrió, lo importante es determinar la validez del descubrimiento.

Una de las ideas que defendieron los que comulgaban con esta propuesta epistemológica era que cuando ocurría un cambio en términos de una teoría o de progreso se debía a causa de una verificación experimental de una "nueva" teoría científica, hecha desde una metodología científica concebida como objetiva y universal.

\section{Nueva filosofía de la ciencia y racionalismo crítico}

En los cuadros 3, 4, 5 y 6 se presentan las ATC's que coinciden con algunos de los planteamientos teóricos en lo que respecta a los criterios denominados: a) unidad estructural del conocimiento, b) encuadre de la actividad científica, c) imagen de ciencia y d) lo procedimental de la ciencia.

Se entiende, desde la propuesta de Popper, que las teorías son conjeturas arriesgadas y poseen un carácter hipotético y revisable (DIÉGUEZ, 2005). Dicha posición epistemológica privilegia el lenguaje formal, es decir, un lenguaje especializado propio de la ciencia, el cual se califica de sintáctico, es decir, lógico-lingüístico, que tiene como objetivo generar análisis rigurosos de la estructura y validez del conocimiento científico.

Otro de los aspectos que caracteriza la propuesta de Popper es la prioridad que le asigna exclusivamente al contexto de justificación y se desconoce la evolución histórica de la 
Cuadro 3. ACT's propias de la nueva filosofía de la ciencia y el racionalismo crítico

\begin{tabular}{|c|c|c|c|}
\hline \multicolumn{2}{|r|}{ Época } & Nueva filosofía de la ciencia y racionalismo crítico & Documento \\
\hline Criterio & $\begin{array}{c}\text { Unidad } \\
\text { estructural } \\
\text { del } \\
\text { conocimiento }\end{array}$ & $\begin{array}{l}\text {...estudios diacrónicos presuponen una visión } \\
\text { radicalmente nueva de la estructura sincrónica o estática } \\
\text { de las teorías científicas. Los principales rasgos de esta } \\
\text { nueva noción de teoría son los siguientes. Las teorías } \\
\text { científicas, en su dimensión sincrónica: (1) Son entidades } \\
\text { sumamente complejas y dúctiles, susceptibles de } \\
\text { evolucionar en el tiempo sin perder su identidad. (2) No son } \\
\text { enunciados o secuencias de enunciados y en un sentido } \\
\text { propio no pueden calificarse de verdaderas o falsas, } \\
\text { aunque con ellas sí se realizan afirmaciones empíricas } \\
\text { verdaderas o falsas. (3) Tienen, al menos, un componente } \\
\text { formal, teórico o conceptual (las leyes o hipótesis) y otro } \\
\text { empírico o aplicativo (los sistemas a que se pretende } \\
\text { aplicar). (4) Cierta parte de cada uno se considera } \\
\text { intocable por decisión metodológica (núcleo). Las teorías } \\
\text { tienen pues partes "esenciales" y partes "accidentales", en } \\
\text { ello radica su ductilidad. (5) Tienen diversos niveles de } \\
\text { empiricidad. (6) Es la parte específica, "accidental" del } \\
\text { formalismo el que recibe el peso de la contrastación. (7) } \\
\text { Llevan asociadas normas, valores, o simplemente } \\
\text { indicaciones, metodológicas y evaluativas, algunas de ellas } \\
\text { fuertemente dependientes del contexto, p. 42-43. }\end{array}$ & DC \\
\hline
\end{tabular}

ciencia. En lo que tiene que ver con la evolución de la ciencia, afirma Popper que dicho proceso se lleva a cabo mediante la falsabilidad de teorías.

Los cambios ocurridos en la filosofía de la ciencia específicamente debido al trabajo de Kuhn y otros como Hanson, Lakatos, Feyerabend, Toulmin y Laudan quienes ponen en duda la distinción tajante entre el contexto de descubrimiento y el contexto de justificación, el problema de la carga teórica de las observaciones y el problema de la inconmensurabilidad entre teorías, las nociones de progreso y racionalidad científica, la relevancia y alcance de los análisis formales y el problema del relativismo, afirmando que la complejidad y riqueza de los elementos involucrados en la ciencia escapa a cualquier intento de formalización (LORENZANO, 2001). Para Kuhn como uno de los representantes más importantes de este pensamiento epistemológico, los factores externalistas en el cambio científico son importantes al momento de saber cómo el conocimiento científico se construye, por tal razón acuden a lo que se denomina contexto de descubrimiento. Los representantes de esta posición epistemológica consideran que una teoría científica posee las siguientes características: a) son identidades complejas con la particularidad de poder evolucionar en el tiempo; b) no son verdades absolutas; c) están conformadas por leyes o hipótesis; d) poseen un aspecto intocable, que por acuerdo en lo metodológico sería lo que se denomina el núcleo de la teoría; e) están relacionadas con el contexto en el que se formulan.

\section{Visiones contemporáneas: Semanticismo}

En los siguientes cuadros (7, 8 y 9), se presentan las ACT's que evidencia un parecido con los planteamientos conceptuales y teóricos correspondientes a la época denominada Visiones 
Cuadro 3. continuação

\begin{tabular}{|c|c|c|c|}
\hline \multicolumn{2}{|r|}{ Época } & Nueva filosofía de la ciencia y racionalismo crítico & Documento \\
\hline Criterio & $\begin{array}{c}\text { Unidad } \\
\text { estructural } \\
\text { del } \\
\text { conocimiento }\end{array}$ & 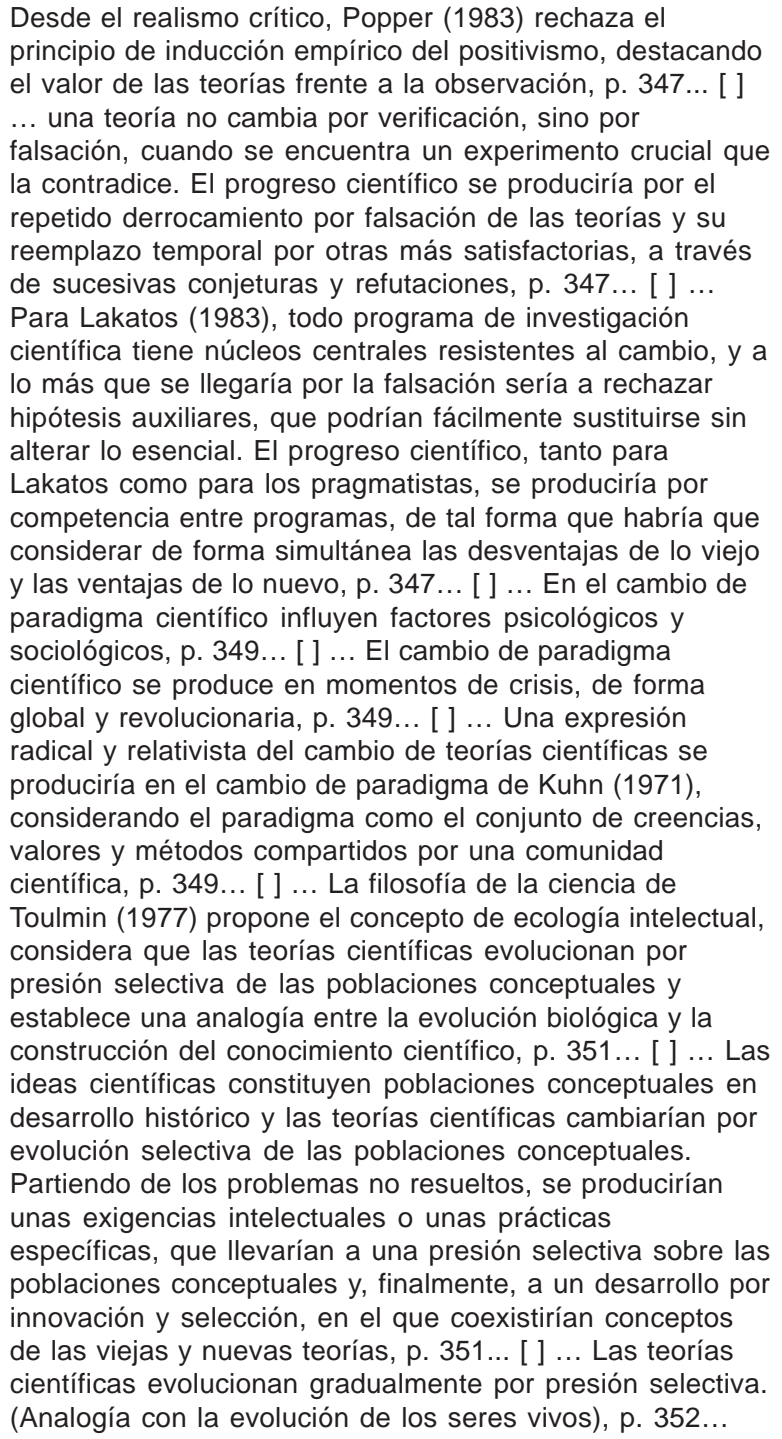 & MJ \\
\hline
\end{tabular}

Fonte: elaborado pelos autores.

contemporáneas; para la organización de la información se formularon los criterios denominados: a) unidad estructural del conocimiento, b) lentes conceptuales, y c) lo procedimental de la ciencia.

Los nuevos planteamientos de los epistemólogos por ejemplo Suppe, Giere y Fraassen, suscitados después de la mitad del siglo XX, proponen que no hay una relación tan directa entre lo que decimos (proposiciones) y los fenómenos (lo que sucede en el mundo), sino que esa relación está mediada por los modelos en tanto representaciones abstractas del mundo, 
Cuadro 4. ACT's propias de la nueva filosofía de la ciencia y el racionalismo crítico

\begin{tabular}{|c|c|l|c|}
\hline \multicolumn{2}{|c|}{ Época } & Nueva filosofía de la ciencia y racionalismo crítico & Documento \\
\hline \multirow{2}{*}{ Criterio } & $\begin{array}{c}\text { Encuadre de } \\
\text { la actividad } \\
\text { científica }\end{array}$ & $\begin{array}{l}\text { f..Kuhn propone el constructo de ejemplar, entendido } \\
\text { como modelo científico a imitar en la búsqueda de nuevas } \\
\text { soluciones a los problemas que van investigándose, } \\
\text { soluciones inspiradas en esa solución canónica y } \\
\text { funcional que se sigue de cerca al hacer ciencia, p. 147... }\end{array}$ & AABmo \\
\cline { 3 - 5 } & $\begin{array}{l}\text { En los períodos de ciencia normal, los científicos trabajan } \\
\text { dentro de un paradigma compartido, solucionando las } \\
\text { anomalías que se producen en el mismo, p. 349... }\end{array}$ & MJ \\
\hline
\end{tabular}

Fonte: elaborado pelos autores.

Cuadro 5. ACT's pertenecientes a la nueva filosofía de la ciencia y el racionalismo crítico

\begin{tabular}{|c|c|l|c|}
\hline \multicolumn{2}{|c|}{ Época } & \multicolumn{1}{|c|}{ Posmodernismo y visiones contemporáneas } & Documento \\
\hline Criterio & $\begin{array}{l}\text { Imagen de } \\
\text { ciencia } \\
\text { pinta la ciencia como un discurso caprichoso, autoritario y } \\
\text { vacío, producto de sórdidas negociaciones entre elites. } \\
\text { Para los relativistas, todas las formas de ver la realidad } \\
\text { serían igualmente válidas y valiosas; con este punto de } \\
\text { partida, se pierde la posibilidad de dar cuenta, desde un } \\
\text { punto de vista epistemológico estricto, de la evolución } \\
\text { histórica ascendente de la ciencia y de sus notables } \\
\text { logros, que el resto de los mortales no osarían poner en } \\
\text { tela de juicio, p. 54... }\end{array}$ & AABme \\
\hline
\end{tabular}

Fonte: elaborado pelos autores.

Cuadro 6. ACT's concernientes a la nueva filosofía de la ciencia y el racionalismo crítico

\begin{tabular}{|c|c|c|c|}
\hline \multicolumn{2}{|r|}{ Época } & Nueva filosofía de la ciencia y racionalismo crítico & Documento \\
\hline \multirow[b]{3}{*}{ Criterio } & \multirow{3}{*}{$\begin{array}{l}\text { Lo } \\
\text { procedimental } \\
\text { de la ciencia }\end{array}$} & $\begin{array}{l}\text {...en el plano metodológico, el discurso relativista se } \\
\text { despeja sin culpas del rígido corsé del método científico, } p \text {. } \\
54 \ldots\end{array}$ & AABme \\
\hline & & $\begin{array}{l}\text {... El cambio de paradigma se produciría en los períodos de } \\
\text { ciencia revolucionaria, en momentos de crisis, más por } \\
\text { reconstrucción del campo que por acumulación o } \\
\text { ampliación del antiguo paradigma, que resultaría } \\
\text { inconmensurable con el nuevo, p. } 349 \ldots\end{array}$ & \multirow[t]{2}{*}{ MJ } \\
\hline & & $\begin{array}{l}\text {... Laudan (1986) establece el concepto de tradiciones } \\
\text { deinvestigación (que incluye supuestos, metodologías, } \\
\text { problemas y teorías) como semejante a los programas de } \\
\text { investigación de Lakatos o a los paradigmas de Kuhn, } \\
\text { aunque el cambio científico se produciría de una forma } \\
\text { continua al resolver los problemas, de manera que la } \\
\text { ciencia progresa sólo si las teorías sucesivas resuelven } \\
\text { más problemas que sus predecesoras. El cambio de } \\
\text { tradición de investigación se daría cuando exista, además, } \\
\text { un cambio ontológico y metodológico, que afectaría a los } \\
\text { supuestos básicos, a la naturaleza de las preguntas, a los } \\
\text { fines y a los valores, p. } 350 . . .\end{array}$ & \\
\hline
\end{tabular}

Fonte: elaborado pelos autores. 
Afirmaciones epistemológicas con "alta carga teórica”...

Cuadro 7. $\mathrm{ACT}^{\prime}$ s tocantes al posmodernismo y visiones contemporáneas

\begin{tabular}{|c|c|c|c|}
\hline \multicolumn{2}{|r|}{ Época } & Posmodernismo y visiones contemporáneas & Documento \\
\hline \multirow{6}{*}{ Criterio } & \multirow{6}{*}{$\begin{array}{l}\text { Unidad } \\
\text { estructural } \\
\text { del } \\
\text { conocimiento } \\
\text { "Modelo }\end{array}$} & $\begin{array}{l}\text {... constituyen el centro de la parte aplicativa de una teoría } \\
\text { y forman una clase o conjunto que queda caracterizado } \\
\text { por las leyes científicas (axiomas en sentido propio) de } \\
\text { esa teoría, p. } 147 \ldots\end{array}$ & \multirow[t]{5}{*}{ AABmo } \\
\hline & & $\begin{array}{l}\text {...son proyecciones de la teoría al mundo, o sus } \\
\text { realizaciones posibles, p. } 147 \ldots\end{array}$ & \\
\hline & & $\begin{array}{l}\text {... una teoría es por tanto una familia de modelos, pero más } \\
\text { que una simple suma de estos modelos, porque estos } \\
\text { últimos están vinculados por relaciones lógicas y } \\
\text { experimentales que aseguran cierta coherencia al } \\
\text { conjunto, p. } 150 \ldots\end{array}$ & \\
\hline & & $\begin{array}{l}\text {...no hay una relación tan directa entre lo que decimos } \\
\text { (proposiciones) y los fenómenos, sino que esa relación } \\
\text { está mediada por los modelos en tanto representaciones } \\
\text { abstractas del mundo, representaciones que no son } \\
\text { reducibles ni a enunciados ni a realidad, p. } 150 . .\end{array}$ & \\
\hline & & $\begin{array}{l}\text {... el modelo teórico se relaciona sustantivamente con dos } \\
\text { elementos: } 1 \text {. El conjunto - amplio y heterogéneo- de } \\
\text { recursos simbólicos (expresivos) que se sirve para } \\
\text { definirlo; y } 2 \text {. El mundo (sistema) al cual viene a modelizar, } \\
\text { con el cual mantiene una relación de parecido que } \\
\text { técnicamente se llama similaridad, p. } 152 \ldots\end{array}$ & \\
\hline & & $\begin{array}{l}\text {.... se considera que el componente más básico para la } \\
\text { identidad de una teoría es una clase de estructuras y más } \\
\text { específicamente una clase de modelos, p. } 13 \ldots . .\end{array}$ & PL \\
\hline
\end{tabular}

continua

representaciones que no son reducibles ni a enunciados ni a realidad (ADURIZ-BRAVO, 2011). Lo anterior se propone ya que la mayoría de las tesis y estudios diacrónicos como por ejemplo: Hanson, Lakatos, Feyerabend, Toulmin, Laudan y especialmente Kuhn, subyace una nueva concepción acerca de la naturaleza y estructura sincrónica de las teorías científicas, que se supone más apegada a la práctica científica tal como la historia nos las presenta; esta nueva noción, a la que los filósofos se refieren con variedad terminológica (paradigma Kuhn; programa de investigación Lakatos; tradición de investigación Laudan), la cual sería imprecisa, en ocasiones de modo tan extremo que termina por desdibujar casi en su totalidad, lo que parecen intuiciones correctas por los y las científicas (Lorenzano, 2001). Suppe, Giere y van Fraseen intentan mostrar que una reinterpretación de las teorías científicas como un conjunto de modelos es más exitosa para una comprensión de la teorización científica y puede proporcionar una imagen más satisfactoria de la relación entre las teorías científicas y el mundo real, a causa del rol mediador de los modelos (LOMBARDI, 1997; ADÚRIZ-BRAVO, 2011).

La comunidad que rechaza los planteamientos epistemológicos de la nueva filosofía de la ciencia (LORENZANO, 2001), parten de la propuesta que una teoría empírica no es una 
Cuadro 7. continuação

\begin{tabular}{|c|c|c|c|}
\hline \multicolumn{2}{|r|}{ Época } & Posmodernismo y visiones contemporáneas & Documento \\
\hline \multirow{7}{*}{ Criterio } & \multirow{7}{*}{$\begin{array}{l}\text { Unidad } \\
\text { estructural } \\
\text { del } \\
\text { conocimiento } \\
\text { "Modelo }\end{array}$} & $\begin{array}{l}\text {...la noción de modelo es una noción fundamentalmente } \\
\text { semántica (algo es modelo de una afirmación si la } \\
\text { afirmación es verdadera de ello), se denomina concepción } \\
\text { semántica a este nuevo enfoque que enfatiza la } \\
\text { importancia de los modelos en el análisis de la ciencia, p. } \\
46 \ldots\end{array}$ & \multirow[t]{7}{*}{ DC } \\
\hline & & $\begin{array}{l}\text {...Un modelo, en su acepción informal mínima, es un } \\
\text { sistema o "trozo de la realidad" constituido por entidades } \\
\text { de diverso tipo que realiza una serie de afirmaciones, las } \\
\text { realiza en el sentido de que en dicho sistema "pasa lo que } \\
\text { las afirmaciones dicen" o, más precisamente, las } \\
\text { afirmaciones son verdaderas en dicho sistema, p. } 46 . .\end{array}$ & \\
\hline & & $\begin{array}{l}\text {... Lo importante es pues qué modelos determina una teoría, } \\
\text { no los recursos lingüísticos que emplea para ello, p. } 48 \ldots\end{array}$ & \\
\hline & & $\begin{array}{l}\text {... De acuerdo con la concepción semántica, presentar } \\
\text { una teoría es presentar una familia de modelos. Esta familia } \\
\text { puede ser descrita de varios modos, mediante enunciados } \\
\text { diferentes en lenguajes diferentes, y ninguna formulación } \\
\text { lingüística tiene ningún estatuto privilegiado, p. } 50 \ldots\end{array}$ & \\
\hline & & $\begin{array}{l}\text {... las leyes definen una serie de modelos significa sólo } \\
\text { que las leyes determinan qué entidades son las que se } \\
\text { comportan de acuerdo con la teoría, p. } 51 \ldots\end{array}$ & \\
\hline & & $\begin{array}{l}\text {... Una teoría determina una clase de modelos para algo, } \\
\text { para dar cuenta de ciertos datos, fenómenos o } \\
\text { experiencias correspondientes a determinado ámbito de la } \\
\text { realidad, p. } 52 \ldots\end{array}$ & \\
\hline & & $\begin{array}{l}\text {...Un modelo teórico es parte de un mundo imaginado, } \mathrm{p} \text {. } \\
73 \ldots\end{array}$ & \\
\hline
\end{tabular}

Fonte: elaborado pelos autores.

Cuadro 8. ACT's correspondientes al posmodernismo y visiones contemporáneas

\begin{tabular}{|c|c|c|c|}
\hline \multicolumn{2}{|c|}{ Época } & Posmodernismo y visiones contemporáneas & Documento \\
\hline Criterio & $\begin{array}{c}\text { Lentes } \\
\text { conceptuales }\end{array}$ & $\begin{array}{l}\text {... Giere defiende sobre estas bases cierto tipo de } \\
\text { "realismo", que él denomina realismo constructivista, p., } \\
75 \ldots \text { La ciencia tiene un aspecto esencialmente } \\
\text { constructivo, la definición de los modelos, y modelos } \\
\text { diferentes pueden ser representaciones alternativas de un } \\
\text { mismo sistema físico. Hay modelos mejores que otros, pero } \\
\text { eso no se puede especificar apelando exclusivamente al } \\
\text { mundo. Nada en el mundo mismo fija los aspectos a } \\
\text { representar, ni cuan buena es la representación. La } \\
\text { especificación debe apelar necesariamente a intereses } \\
\text { humanos, no sólo epistémicos o científicos, sino también a } \\
\text { prácticos de diverso tipo. Eso supone una cierta dosis de } \\
\text { relativismo, pero no es un relativismo radical, p. } 75 \ldots\end{array}$ & DC \\
\hline
\end{tabular}

Fonte: elaborado pelos autores. 
Afirmaciones epistemológicas con "alta carga teórica"...

Cuadro 9. $\mathrm{ACT}^{\prime}$ s referentes al posmodernismo y visiones contemporáneas

\begin{tabular}{|c|c|l|c|}
\hline \multicolumn{2}{|c|}{ Época } & \multicolumn{1}{c|}{ Posmodernismo y visiones contemporáneas } & Documento \\
\hline Criterio & $\begin{array}{c}\text { Lo } \\
\text { procedimental } \\
\text { de la ciencia }\end{array}$ & $\begin{array}{l}\text {..Una concepción moderada de la metodología de la ciencia } \\
\text { pone el foco en la capacidad de pensar acerca del mundo } \\
\text { con modelos teóricos, de generar lenguajes abstractos para } \\
\text { crear y comunicar conocimiento, y de intervenir } \\
\text { transformadoramente sobre los fenómenos, p. 55... }\end{array}$ & AABme \\
\hline
\end{tabular}

Fonte: elaborado pelos autores.

entidad lingüística, más concretamente no es solo un conjunto de enunciados axiomáticos o una conjunción de ellos, se considera que el componente más básico para la identidad de una teoría es una clase de estructuras y más específicamente una clase de modelos, que pretenden dar cuenta o representar, de manera más o menos idealizada o aproximada, cierto datos, fenómenos o experiencias correspondientes a determinado ámbito de la realidad (LORENZANO, 2001). Adúriz-Bravo (2011) propone que los representantes de este pensamiento epistemológico como Suppe, Giere y van Fraassen suponen que las teorías no son sólo los enunciados teóricos de los que aquellas están compuestas, sino que contienen también los hechos interpretados por ellas, así una teoría es por tanto una familia de modelos, pero más que una simple suma de estos modelos, porque estos últimos están vinculados por relaciones lógicas y experimentales que aseguran cierta coherencia al sistema.

Esta propuesta epistemológica se ha denominado concepción semántica de las teorías científicas, y los miembros de este movimiento comparten el espíritu formalista del positivismo lógico aunque no la letra (LORENZANO, 2001), es decir, de acuerdo con esta concepción semántica, presentar una teoría es presentar una familia de modelos. Esta familia puede ser descrita de varios modos, mediante enunciados diferentes en lenguajes diferentes, y ninguna formulación lingüística tiene ningún estatuto privilegiado. Específicamente, no se atribuye ninguna importancia a la axiomatización como tal, e incluso la teoría podría no ser axiomatizable (DIEZ, 1997).

Diez (1997) define la categoría de modelo como un sistema o "trozo de la realidad" constituido por entidades de diverso tipo que realiza una serie de afirmaciones, las realiza en el sentido de que en dicho sistema "pasa lo que las afirmaciones dicen” o, más precisamente, las afirmaciones son verdaderas en dicho sistema. Para Adúriz-Bravo (2011), los modelos son proyecciones de la teoría al mundo, o sus realizaciones posibles, es decir, un modelo es un sistema que se expresa formalmente mediante una secuencia de conjuntos, el primero contiene los individuos del sistema y los restantes son relaciones y funciones entre dichos individuos (DIEZ, 1997). Adúriz-Bravo (2011), acude a los planteamientos de Giere, quien propone la categoría de modelo teórico. Los modelos teóricos se relacionan sustantivamente con dos elementos: 1 . El conjunto -amplio y heterogéneo- de recursos simbólicos (expresivos) que se sirve para definirlo; y 2. El mundo (sistema) al cual viene a modelizar, con el cual mantiene una relación de parecido que técnicamente se llama similaridad, de otra parte, Giere expone que una teoría esta compuesta de dos elementos: 1. una población de modelos, y 2. varias hipótesis conectando esos modelos con sistemas en el mundo real (DIEZ, 1997); lo que lleva a pensar que para Giere un modelo teórico es parte de un mundo imaginado. No existe en ningún lugar excepto en las mentes de las y los científicos. 
Para Giere las hipótesis teóricas son el vehículo del conocimiento científico para hacer aserciones con contenidos empíricos sobre la realidad: ellas afirman que el modelo se parece al sistema en tales y cuales aspectos y con tales y cuales grados de ajuste. Estas hipótesis son, por tanto, susceptibles de prueba y, consecuentemente, corroborables o refutables de manera parcial y aproximativa durante la investigación científica (ADÚRIZ-BRAVO, 2011).

Una hipótesis teórica se comprende como un enunciado o proposición que afirma cierto tipo de relación entre un modelo y un sistema real determinado (DIEZ, 1997). Estableciendo que a diferencia de los modelos, las hipótesis teóricas sí son entidades lingüísticas (preposicionales), verdaderas o falsas, la relación que se afirma en la hipótesis teórica no es la de identidad, no se afirma que cierto sistema es el modelo; dado que los sistemas son entidades físicas y los modelos no lo son, son entidades abstractas. La relación sería la de similaridad. Pero toda relación de similaridad debe ser cualificada para ser mínimamente precisa. Debe restringirse a determinados aspectos y, en ellos, a cierto grado. La forma general de la hipótesis teórica es pues la siguiente: "Tal sistema real identificable es similar al modelo designado en los aspectos y grados indicados".

Por lo que se propone que una teoría determina una clase de modelos para algo, para dar cuenta de ciertos datos, fenómenos o experiencias correspondientes a determinado ámbito de la realidad. Parte de la identificación de la teoría consiste entonces en la identificación de esos fenómenos empíricos de los que pretende dar cuenta (DÍEZ, 1997).

\section{Ideas centrales de los hallazgos}

En los cuadros 10, 11 e 12, se muestran encuentros teóricos generales que presentan los autores en los textos analizados.

En el Cuadro 10 se presentan aquellas afirmaciones que reúnen las ideas centrales de los autores con respecto a la categoría de unidad estructural del conocimiento, se evidencia que los acuerdos están centrando en considerar a la teoría como el objeto de estudio y justificación del desarrollo del conocimiento científico, en la que se le considera como un conjunto de enunciados los cuales están organizados axiomáticamente, siendo lo más importante para comprender qué es la ciencia y cómo evoluciona.

En lo que respecta a la época de la nueva filosofía de la ciencia y racionalismo crítico, se encontró que lo más importante para comprender el desarrollo del conocimiento son los factores externalistas, dado que lo primordial no es buscar la formalización del conocimiento,

Cuadro 10. Acuerdos conceptuales hallados

\begin{tabular}{|c|c|c|}
\hline \multicolumn{2}{|c|}{ Época } & \multicolumn{1}{c|}{$\begin{array}{c}\text { Positivismo lógico y concepcion heredada } \\
\text { Hallazgos generales }\end{array}$} \\
\hline Criterio & $\begin{array}{c}\text { Unidad } \\
\text { estructural } \\
\text { del } \\
\text { conocimiento }\end{array}$ & $\begin{array}{l}\text { Los acuerdos conceptuales que se obtuvieron se podrían especificar en } \\
\text { dos apartados: } \\
\text { 1. Las teorías empíricas asumidas como conjuntos de enunciados } \\
\text { organizados axiomáticamente. } \\
\text { 2. Una teoría es una clase de axiomas, lo que reafirma el énfasis en lo } \\
\text { sintáctico en esta época de la epistemología. }\end{array}$ \\
\hline
\end{tabular}

Fonte: elaborado pelos autores. 
sino comprender los factores que posibilitan que las comunidades científicas generen las interpretaciones de los fenómenos científicos, lo anterior se muestra en el Cuadro 11.

Cuadro 11. Acuerdos conceptuales hallados

\begin{tabular}{|c|c|c|}
\hline \multicolumn{2}{|c|}{ Época } & \multicolumn{1}{c|}{$\begin{array}{c}\text { Nueva filosofía de la ciencia y racionalismo crítico } \\
\text { Hallazgos generales }\end{array}$} \\
\hline Criterio & $\begin{array}{c}\text { Conocimiento } \\
\text { científico }\end{array}$ & $\begin{array}{l}\text { Hechos los análisis se encontraron los siguientes acuerdos: } \\
\text { - Se piensa que desde los planteamientos de los representantes de esta } \\
\text { época epistemológica, la ciencia es analizada desde una posición } \\
\text { sincrónica lo que permite pensar por ejemplo, que todas las formas de } \\
\text { percibir la realidad por parte de los y las científicas tendrían el mismo valor, } \\
\text { como también se puede inferir que las teorías no son enunciados o } \\
\text { sucesiones de enunciados, desde los planteamientos de los relativistas, no } \\
\text { se puede afirmar cuales son verdaderas o falsas. }\end{array}$ \\
\hline
\end{tabular}

Fonte: elaborado pelos autores.

Hechas las críticas a las anteriores épocas epistemológicas, los/as epistemólogos/as que no concuerdan con estas posturas, acuden a planteamientos teóricos en el que afirman que la unidad estructural del conocimiento científico no sería la teoría sino la de modelo teórico, afirmando que el conocimiento científico no está compuesto por un conjunto de enunciado axiomáticos, sino que por el contrario estaría conformado por una clase de modelos, los cuales pretenden representan aproximadamente la realidad; en el Cuadro 12, se exponen algunos de los consenso encontrados en los textos analizados.

Cuadro 12. Acuerdos conceptuales hallados

\begin{tabular}{|c|c|l|}
\hline \multicolumn{2}{|c|}{ Época } & \multicolumn{1}{c|}{$\begin{array}{c}\text { Posmodernismo y visiones contemporáneas } \\
\text { Similitudes }\end{array}$} \\
\hline Criterio & $\begin{array}{c}\text { Unidad } \\
\text { estructural } \\
\text { del } \\
\text { conocimiento } \\
\text { "Modelo }\end{array}$ & $\begin{array}{l}\text { Los textos analizados concuerdan en que: } \\
\text { - Los modelos son los componentes más básicos para la identidad de una } \\
\text { teoría y a su vez la parte aplicativa de las mismas, siendo una clase de } \\
\text { estructuras y más específicamente una clase de modelos, los cuales dan } \\
\text { cuenta de ciertos datos, fenómenos o experiencias correspondientes a un } \\
\text { determinando trozo de la realidad. } \\
\text { - La idea de modelo desde la concepción semántica es interpretada como } \\
\text { una teoría que está constituida por una familia de modelos y la cual se } \\
\text { puede describir por medio de enunciados diferentes en lenguaje diferentes, } \\
\text { pero lo más importante es qué modelos determinan la teoría y no los } \\
\text { recursos lingüísticos que se emplean para esto. }\end{array}$ \\
\hline
\end{tabular}

Fonte: elaborado pelos autores.

\section{Inferencias finales}

El estudio bibliográfico que hemos acometido nos permitió identificar aquellas afirmaciones con una alta carga teórica (ATC's) que los especialistas formulan para caracterizar diferentes conceptualizaciones epistemológicas producidas en el siglo XX, encontrando puntos de acuerdos con respecto a lo que comprenden por el desarrollo del conocimiento científico, 
como también los problemas que presentan las posturas epistemológicas que se desarrollaron a comienzos del siglo XX y a mitad del mismo.

Para solventar los inconvenientes teóricos de dichas posturas epistemológicas, los especialistas han generado algunas propuestas innovadoras para comprender el desarrollo del conocimiento; en este documento se ha acudido a una de ellas, denominada concepción semántica. La elección se ha hecho porque consideramos que es la más potente para relacionarla con los desarrollos teóricos y procedimentales de la didáctica de las ciencias y de la naturaleza de la ciencia, generando elementos teóricos innovadores para dichos campos de conocimiento (DEVELAKI, 2007; KOPONEN, 2007; ERDURAN; DUSCHL, 2004; IZQUIERDO, 2000).

Consideramos que dicho análisis permite a los y las integrantes de la comunidad en didáctica establecer con mayor rigurosidad los problemas que presentan las viejas concepciones epistemológicas y cómo los “nuevos" planteamientos epistemológicos dan un mayor poder explicativo al desarrollo del conocimiento y su relación con otras ciencias, por ejemplo la didáctica de las ciencias.

\section{Referencias}

ADÚRIZ-BRAVO, A. Concepto de modelo científico: una mirada epistemológica de su evolución. In: GALAGOVSKY, L. (Coord.). Didáctica de las ciencias naturales: el caso de los modelos científicos. Buenos Aires: Lugar Editorial, 2011. p. 141-161.

• ¿Existirá el “método científico”? In: GALAGOVSKY, L. (Coord.). ¿Qué tienen de "naturales" las ciencias naturales. Buenos Aires: Biblos, 2008. p. 47-59.

ADÚRIZ-BRAVO, A.; IZQUIERDO-AYMERICH, M. Un modelo de modelo científico para la enseñanza de las ciencias naturales. Revista Electrónica de Investigación en Educación en Ciencias. v. 4, número especial, p. 40-49, 2009. Disponible en: <http:// www.exa.unicen.edu.ar/ reiec/files/num_esp/2009/REIEC_esp_2009_art4.pdf>. Acceso en: Marzo 2011

ADÚRIZ-BRAVO, A.; IZQUIERDO-AYMERICH, M.; ESTANY, A. Una propuesta para estructurar la enseñanza de la filosofía de la ciencia para el profesorado de ciencias en formación. Enseñanza de las Ciencias, Barcelona, v. 20, n. 3, p. 465- 476, 2002.

AMADOR-RODRÍGUEZ, R. Y.; ADÚRIZ-BRAVO, A. Una postura teórica para analizar la interacción entre la naturaleza de la ciencia y la didáctica de las ciencias. Avances en educación en ciencia y tecnología: Enfoques y estrategias. Catamarca: Universidad Nacional de Catamarca, 2009.

ARIZA, A. Y.; ADÚRIZ-BRAVO, A.; LORENZANO, P. Algunos aspectos de interés sobre la periodización de la epistemología con finalidad didáctica. Debates, reflexiones e interrogantes en la educación en ciencias. Catamarca, Universidad Nacional de Catamarca, Primera Edición, p. 19-20, 2009. 
DEVELAKI, M. The model-based view of scientific theories and the structuring of school science programmes. Science \& Education, v. 7, n. 16, p. 725-749, 2007.

DIÉGUEZ, A. Filosofía de la ciencia. Editorial biblioteca nueva. España, 2005.

DÍEZ CALZADA, J. A. La concepción semántica de las teorías científicas. Éndoxa: Series Filosóficas, UNED, Madrid, n. 8, p. 41-91, 1997.

ECHEVARRÍA, J. Filosofía de la Ciencia, Ediciones Akal S.A. Madrid-España, 1998.

ERDURAN, S.; DUSCHL, R. Interdisciplinary characterizations of models and the nature of chemical knowledge in the classroom. Studies in Science Education, n. 40, p. 111-144, 2004.

IZQUIERDO-AYMERICH, M. Fundamentos epistemológicos. In: PERALES, F. J.; CAÑAL, P. (Comp.). Didáctica de las ciencias experimentales: teoría y práctica de la enseñanza de las ciencias, Alcoy: Marfil. p. 35-64, 2000.

KOPONEN, I. T. Models and modelling in physics education: A critical re-analysis of philosophical underpinnings and suggestions for revisions. Science \& Education, v. 7-8, n. 16, p. 751-773, 2007.

LEDERMAN, N. G. Students' and teachers' conceptions of the nature of science: a review of the research. Journal of Research in Science Teaching, v. 4, n. 29 , 331-359, 1992.

LOMBARDI, O. La noción de modelo en ciencias. Educación en Ciencias, v. 2, n. 4 , p. 4-13, 1997.

LORENZANO, P. La teorización filosófica sobre la ciencia en el siglo XX. Boletín de la Biblioteca del Congreso de la Nación, n. 121, p. 29-43, 2001. En: http:// www.infoamerica.org/teoria_articulos/lorenzano01.pdf. Acceso Marzo 2011.

McCOMAS, W. The nature of science in science education: rationales and strategies. Dordrecht: Kluwer, 1998.

MELLADO JIMÉNEZ, V. Cambio didáctico del profesorado de ciencias experimentales y filosofía de la ciencia. Enseñanza de las ciencias, Barcelona, v. 21, n. 3, p. 343-358, 2003. 